\title{
Influence of the length of target DNA overhang proximal to the array surface on discrimination of single-base mismatches on a 25 -mer oligonucleotide array
}

\author{
Jenny Tomlinson ${ }^{1 *}$, Catherine Harrison ${ }^{1}$, Neil Boonham ${ }^{1}$, Sarah A Goodchild ${ }^{2}$ and Simon A Weller ${ }^{2}$
}

\begin{abstract}
Background: The performance of probes on an oligonucleotide microarray can be characterised in terms of hybridisation signal strength and the ability to discriminate sequence mismatches between the probe and the hybridising target strand, such as those resulting from SNPs. Various properties of the probe affect mismatch discrimination, such as probe length and the position of mismatched bases, and the effects of these factors have been well characterised in a variety of array formats.

Results: A low-density microarray was developed to systematically investigate the effect of a probe's position within hybridised target PCR products on the tolerance and discrimination of single-nucleotide mismatches between the probe and target. In line with previous reports, hybridisation signals were attenuated by different degrees depending on the identity of the mismatch, the position of the mismatch within the probe, and the length of the PCR product. However, the same mismatch caused different degrees of attenuation depending on the position of the probe within the hybridising product, such that improved mismatch discrimination was observed for PCR products where a greater proportion of the total length was proximal to the array surface.

Conclusions: These results suggest that the degree of mismatch discrimination can be influenced by the choice of PCR primers, providing a means by which array performance could be fine-tuned in addition to manipulation of the properties of the probes themselves.
\end{abstract}

Keywords: Oligonucleotide microarray, Mismatch discrimination, SNP

\section{Background}

High-density microarrays in a conventional glass slide format with fluorescence detection, such as those used for gene expression studies, pose a relatively high operational burden due to time-consuming protocols and the cost of the equipment required for high-resolution fluorescence detection. In the context of pathogen detection, however, the requirement is more likely to be for a method which allows large numbers of samples to be tested efficiently at one time. Low-density microarrays, such as those using the ArrayTube (AT) platform [1-3]

\footnotetext{
* Correspondence: jenny.tomlinson@fera.gsi.gov.uk

${ }^{1}$ The Food and Environment Research Agency, Sand Hutton, YO41 1LZ York, UK

Full list of author information is available at the end of the article
}

allow the detection of a smaller number of targets (typically $<100$ ) with improved simplicity of handling and lowcost scanning instrumentation.

A typical low-density array protocol involves subjecting sample DNA to multiplex PCR with labelled nucleotides or primers, followed by application of the labelled products to the array and identification of the probes to which the products have hybridised. A frequently used approach is to select conserved target genes, enabling amplification using broad-range PCR primers followed by discrimination of different products by hybridisation to specific probes [4]. Conversely, in order to reliably detect sequence variants (for example, different viral strains or novel viruses) it can be necessary to tolerate sequence differences both in the PCR primers and

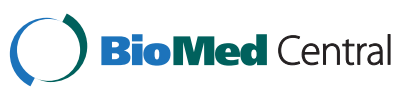


the probes on the array [5]. In order to detect diverse targets (for example, bacterial and viral pathogens) on a single array, it may therefore be necessary to maximise mismatch discrimination for some targets while tolerating mismatches for others.

The performance of probes on an array is affected by factors including probe length [6,7], number and distribution of mismatches between the probe and target [8-10], orientation of attachment to the array surface and presence or absence of spacers [11,12]. The effects of mismatched bases on probe-target hybridisation have been studied in great detail, using microarrays in a variety of formats $[10,13]$. Greater specificity is achieved by using shorter probes [7], but longer probes are associated with higher hybridisation signals [6]. Furthermore, mismatches located at the centre of the probe have a greater effect on hybridisation signals than mismatches close to the ends of the probe $[8,13,14]$. Hybridisation is also dependent on the properties of the target nucleic acid; in particular, relatively long targets are associated with secondary structure which can significantly hinder hybridisation [15-17]. The performance of the array is therefore also influenced by the PCR primers used, in terms of the specificity they confer, their general suitability for PCR (since array sensitivity is largely determined by the sensitivity of the PCR) and the secondary structure of the amplified product. Generalisations can be made about favourable properties of the PCR products, such as length [10] and labelling method, for optimal performance of an array in terms of signal strength, specificity and limit of detection. The location of the PCR primers within the target sequence will also determine the position of the probe within the amplified product. Peytavi et al. [18] reported that the position of the probe can affect hybridisation signal strength; while Stedtfeld at al [19] found that false positive signals can be caused by interaction between overhanging target nucleic acid and labelled background DNA. We hypothesise that the position of the probe within the PCR product (i.e. it's proximity to the $5^{\prime}$ or $3^{\prime}$ end of the hybridising strand) could also influence its performance in terms of the tolerance or discrimination of mismatches. A 25-mer oligonucleotide array in an AT format was designed to investigate this hypothesis.

\section{Methods}

\section{Probe design}

Perfect match probes with a length of 25 bases and a melting temperature of approx. $62^{\circ} \mathrm{C}$ were designed to target Burkholderia pseudomallei chromosome II (accession number CP000571). They were selected in regions of the sequence (Table 1) such that single-base changes could be introduced to result in eight mismatches between the probe and the target sequence (AA, AG, GG,
CC, AC, CT, TT, and GT) at three different positions (in the $3^{\prime}$ third, the middle third, and the $5^{\prime}$ third). In this way, sets of 25 probes were constructed: a perfect match probe and 24 mismatch probes each containing a single mismatch with the target sequence. Microarrays were manufactured by Alere (Jena, Germany) using their ArrayTube platform and consisted of oligonucleotide probes with a 3' amino modification and C6 spacer. Probes were spotted in duplicate.

\section{PCR primer design}

PCR primers were designed based on B. pseudomallei sequence (accession number CP000571) to generate PCR products of different lengths and in different positions relative to the probes. The PCR products generated by different primer pairs could be characterised in terms of the total length of the PCR product and the length of the section of the PCR product overhanging the probe sequence at its $3^{\prime}$ end, referred to as the $5^{\prime}$ segment of the product (see Figure 1); this is the region referred to as the 'surface-proximal tail' by Stedtfeld et al. [19]. Primers were synthesised by MWG Eurofins (Ebersberg, Germany). Their sequences are shown in Table 2.

\section{DNA extraction}

Burkholderia pseudomallei CLO2 DNA was prepared and quanitified by the Defence Science and Technology Organisation (DSTO) in Melbourne, Australia. The DNA extract was sterility checked to enable work under Biological Safety Level 2 (BSL2) conditions.

\section{PCR with biotin labelling}

PCR was carried out in $50 \mu \mathrm{l}$ reactions containing $1 \times$ GoTaq Flexi Colourless Buffer (Promega, Madison, WI, USA); $1.5 \mathrm{mM} \mathrm{MgCl}$; $80 \mu \mathrm{M}$ each dATP, dCTP and dGTP; $52 \mu \mathrm{M}$ dTTP; $28 \mu \mathrm{M}$ biotin-11-dUTP (Thermo Scientific, Waltham, MA, USA); $300 \mathrm{nM}$ forward primer; $300 \mathrm{nM}$ reverse primer; 1.25 units Go Taq polymerase (Promega) and $1 \mathrm{ng}$ B. pseudomallei DNA. Primer combinations were as shown in Table 2. Cycling conditions were $95^{\circ} \mathrm{C}$ for $2 \mathrm{~min}$, followed by 30 three-step cycles of $95^{\circ} \mathrm{C}$ for $30 \mathrm{~s} 58^{\circ} \mathrm{C}$ for $30 \mathrm{~s}$ and $72^{\circ} \mathrm{C}$ for $1 \mathrm{~min}$, with a final extension step at $72^{\circ} \mathrm{C}$ for $5 \mathrm{~min}$. After amplification, reactions were purified using the QIAquick PCR Purification kit (Qiagen, Hilden, Germany) according to the manufacturer's instructions and eluted in nuclease-free water. Approximate DNA concentrations of the purified PCR products were determined using a Qubit fluorometer and dsDNA HS Assay Kit (Life Technologies, Foster City, CA, USA) according to the manufacturer's instructions. Approximate copy numbers were estimated on the basis of DNA concentration and the molecular weight of the predicted product. Each primer combination was initially tested in unlabelled PCR with dNTPs at $80 \mu \mathrm{M}$ each and 
Table 1 Probe sequences

\begin{tabular}{|c|c|}
\hline Probe name & Sequence $\left(5^{\prime}\right.$ to $\left.3^{\prime}\right)$ \\
\hline PM 1 & ACTACGACACACATGACATGATCAA \\
\hline $5 A A 1$ & ACAACGACACACATGACATGATCAA \\
\hline MAA 1 & ACTACGACACACAAGACATGATCAA \\
\hline 3AA 1 & ACTACGACACACATGACATGAACAA \\
\hline $5 A G 1$ & ACTAAGACACACATGACATGATCAA \\
\hline MAG 1 & ACTACGACACAAATGACATGATCAA \\
\hline 3AG 1 & ACTACGACACACATGACATGATAAA \\
\hline 5GG 1 & ACTAGGACACACATGACATGATCAA \\
\hline MGG 1 & ACTACGACACAGATGACATGATCAA \\
\hline 3GG 1 & ACTACGACACACATGACATGATGAA \\
\hline $5 C C 1$ & ACTACCACACACATGACATGATCAA \\
\hline MCC 1 & ACTACGACACACATCACATGATCAA \\
\hline 3CC 1 & ACTACGACACACATGACATCATCAA \\
\hline 5 AC 1 & ACTACAACACACATGACATGATCAA \\
\hline MAC 1 & ACTACGACACACATAACATGATCAA \\
\hline $3 \mathrm{AC} 1$ & ACTACGACACACATGACATAATCAA \\
\hline $5 \mathrm{CT} 1$ & ACTACTACACACATGACATGATCAA \\
\hline MCT 1 & ACTACGACACACATTACATGATCAA \\
\hline 3CT 1 & ACTACGACACACATGACATTATCAA \\
\hline $5 \pi T 1$ & ACTTCGACACACATGACATGATCAA \\
\hline MTT 1 & ACTACGACACACTTGACATGATCAA \\
\hline $3 \pi T 1$ & ACTACGACACACATGACATGTTCAA \\
\hline 5GT 1 & ACTGCGACACACATGACATGATCAA \\
\hline MGT 1 & ACTACGACACACGTGACATGATCAA \\
\hline 3GT 1 & ACTACGACACACATGACATGGTCAA \\
\hline PM 2 & ACGATATCCTCGAAAAGACGATCAA \\
\hline $5 \mathrm{AA} 2$ & ACGAAATCCTCGAAAAGACGATCAA \\
\hline MAA 2 & ACGATATCCACGAAAAGACGATCAA \\
\hline 3AA 2 & ACGATATCCTCGAAAAGACGAACAA \\
\hline $5 A G 2$ & AAGATATCCTCGAAAAGACGATCAA \\
\hline MAG 2 & ACGATATCCTAGAAAAGACGATCAA \\
\hline $3 A G 2$ & ACGATATCCTCGAAAAGACGATAAA \\
\hline $5 G G 2$ & AGGATATCCTCGAAAAGACGATCAA \\
\hline MGG 2 & ACGATATCCTGGAAAAGACGATCAA \\
\hline $3 G G 2$ & ACGATATCCTCGAAAAGACGATGAA \\
\hline $5 C C 2$ & ACCATATCCTCGAAAAGACGATCAA \\
\hline MCC 2 & ACGATATCCTCCAAAAGACGATCAA \\
\hline $3 C C 2$ & ACGATATCCTCGAAAAGACCATCAA \\
\hline 5 AC 2 & ACAATATCCTCGAAAAGACGATCAA \\
\hline MAC 2 & ACGATATCCTCAAAAAGACGATCAA \\
\hline $3 \mathrm{AC} 2$ & ACGATATCCTCGAAAAGACAATCAA \\
\hline $5 \mathrm{CT} 2$ & ACTATATCCTCGAAAAGACGATCAA \\
\hline MCT 2 & ACGATATCCTCTAAAAGACGATCAA \\
\hline 3СТ 2 & ACGATATCCTCGAAAAGACTATCAA \\
\hline
\end{tabular}

Table 1 Probe sequences (Continued)

\begin{tabular}{ll}
\hline $5 \pi T 2$ & ACGTTATCCTCGAAAAGACGATCAA \\
MTT 2 & ACGATATCCTCGTAAAGACGATCAA \\
$3 \pi T 2$ & ACGATATCCTCGAAAAGACGTTCAA \\
$5 G T 2$ & ACGGTATCCTCGAAAAGACGATCAA \\
MGT 2 & ACGATATCCTCGGAAAGACGATCAA \\
$3 G T 2$ & ACGATATCCTCGAAAAGACGGTCAA
\end{tabular}

Mismatched bases are indicated in bold.

the products analysed by agarose gel electrophoresis to confirm the approximate size of the product and absence of primer dimers or other amplification artifacts (data not shown).

\section{Asymmetric PCR with biotin labelling}

Asymmetric PCR is often used to generate predominantly single-stranded PCR products for hybridisation to microarrays, and the presence of the complementary, non-hybridising strand has been shown to influence hybridisation efficiency and other factors $[18,20]$. In order to investigate the effect on tolerance and discrimination of mismatches, forward primer for4 was modified for asymmetric PCR taking into account design considerations for Linear-After-The-Exponential (LATE) PCR [21] as shown in Table 2. PCR reactions and cycling conditions were the same as those used for symmetric PCR, except that the concentration of the reverse (excess) primer was increased to $500 \mathrm{nM}$ and the concentration of the modified forward (limiting) primer was reduced to

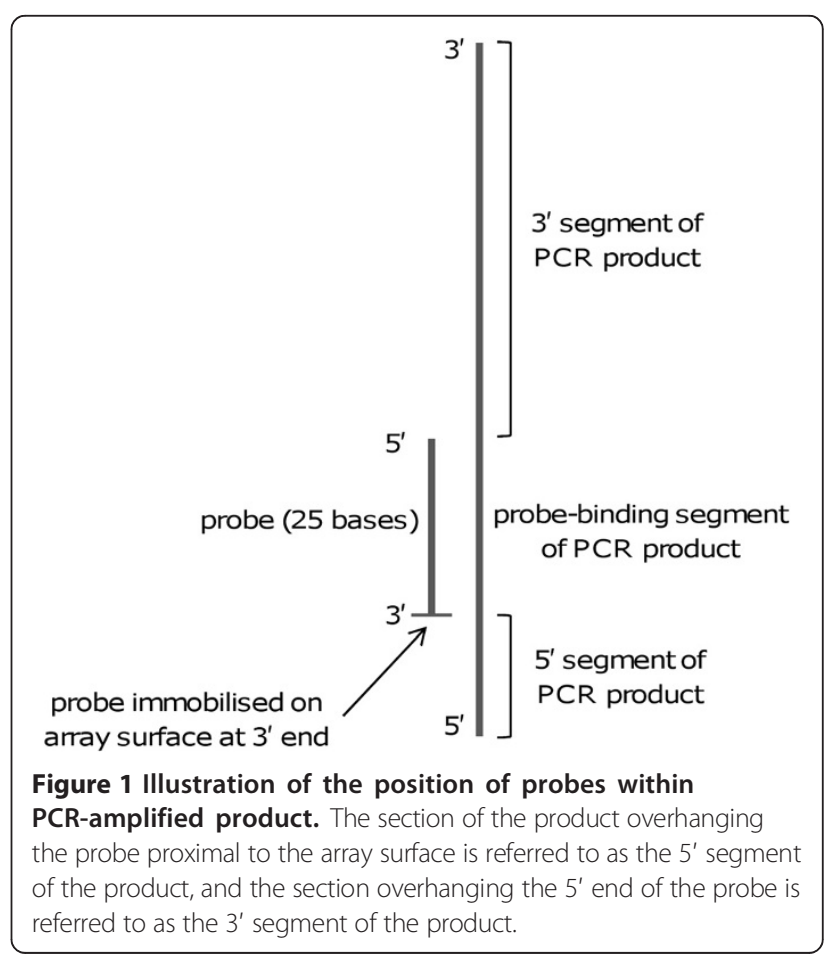


Table 2 Primers used for biotin-labelled PCR

\begin{tabular}{|c|c|c|c|c|c|}
\hline Forward primer & $\begin{array}{l}\text { Forward primer } \\
\left.\text { sequence (5' to } 3^{\prime}\right)\end{array}$ & Reverse primer & $\begin{array}{l}\text { Reverse primer } \\
\left.\text { sequence (5' to } 3^{\prime}\right)\end{array}$ & Amplicon length (bases) & $\begin{array}{l}\text { Length of } 5^{\prime} \\
\text { segment (bases) }\end{array}$ \\
\hline for1 & CTGGAGGAGCCTTCCCTAC & rev1 & GTCGCGACTGCCGCTC & 94 & 43 \\
\hline for2 & CCTCGCATTGCTCAAGCACA & rev1 & as above & 145 & 43 \\
\hline for3 & CGCGACACGCCCATGT & rev1 & as above & 243 & 43 \\
\hline for4 & GCGGACATCGACGGCTATGT & rev1 & as above & 312 & 43 \\
\hline for4 lim* & AACGCGCGGACATCGACGGCTATGT & rev1 & as above & 317 & 43 \\
\hline for5 & GCGCATACGATTCTTCAAAAGGC & rev1 & as above & 978 & 43 \\
\hline for3 & as above & rev2 & ACGCCTTACGTATCGGATCG & 298 & 107 \\
\hline for6 & CTTCGGGCCACCGCTACTA & rev3 & TGTGCTGTGGGCCGTGCT & 300 & 149 \\
\hline for1 & as above & rev4 & TCACCGCGCCTTGCTCGT & 242 & 191 \\
\hline for2 & as above & rev4 & as above & 293 & 191 \\
\hline for3 & as above & rev4 & as above & 382 & 191 \\
\hline for7 & as above & rev4 & as above & 624 & 191 \\
\hline for5 & as above & rev4 & as above & 1126 & 191 \\
\hline for1 & as above & rev5 & CCTCGGGCGTTTCGATCAC & 323 & 272 \\
\hline for4 & as above & rev5 & as above & 541 & 272 \\
\hline for8 & as above & rev5 & as above & 878 & 272 \\
\hline for5 & as above & rev5 & as above & 1027 & 272 \\
\hline for8 & CCAATGCATGTCGGCTCGC & rev6 & ACACCTCTTGCACGGAACCG & 296 & $23^{* *}$ \\
\hline forg & GACACAAGCCGCGAACTGAC & rev7 & CCCAATGTCCGACATAGCC & 295 & $95^{* *}$ \\
\hline for10 & GAGCATCTTCGCGCCATAG & rev8 & TGTCGCGCTCGTTCGCTG & 289 & $149^{* *}$ \\
\hline for7 & CCTTTGCCGTCAGCTTCCG & rev9 & TAGTAGCGGTGGCCCGAAG & 301 & $201^{* *}$ \\
\hline for11 & CAGTTGTCCCTGAAGCGCCT & rev10 & TTGAGCAATGCGAGGCTGC & 317 & $246^{* *}$ \\
\hline
\end{tabular}

${ }^{*}$ Modified for asymmetrical (Linear-After-The-Exponential) PCR.

**Probe set 2 - all other primer combinations for probe set 1.

25,50 or $100 \mathrm{nM}$ to give primer ratios of $20: 1,10: 1$ and 5:1, respectively. Approximate total DNA concentrations (single- and double-stranded DNA) were determined for symmetrical and asymmetrical PCR products using a Qubit fluorometer and ssDNA Assay Kit (Life Technologies) according to the manufacturer's instructions, and concentrations were adjusted to give approximately equal total DNA concentrations. The symmetrical and asymmetrical PCR products were treated with S1 nuclease and subjected to agarose gel electrophoresis to determine the presence or absence of single-stranded amplification products [22]. For treatment with S1 nuclease, $10 \mu \mathrm{l} \mathrm{PCR} \mathrm{prod-}$ uct was combined with 20 units S1 nuclease and $4 \mu \mathrm{l} 5 \times$ S1 nuclease reaction buffer (Thermo Scientific) in a final reaction volume of $20 \mu \mathrm{l}$ and incubated at $37^{\circ} \mathrm{C}$ for $30 \mathrm{~min}$. Treated and untreated PCR products $(10 \mu \mathrm{l}$ and $5 \mu \mathrm{l}$, respectively) were analysed by electrophoresis in $1.2 \%$ agarose gels containing GelRed nucleic acid stain (Biotium, Hayward, CA) at a final concentration of $0.5 \times$.

\section{Array hybridisation and analysis}

Biotin-labelled PCR products were made up to a volume of $40 \mu \mathrm{l}$ in nuclease-free water and mixed with $70 \mu \mathrm{l}$
Nexterion Hybridisation Buffer (Schott, Mainz, Germany), then heated to $95^{\circ} \mathrm{C}$ for $4 \mathrm{~min}$ and cooled to $55^{\circ} \mathrm{C}$. ArrayTubes were conditioned by applying $500 \mu \mathrm{l}$ nuclease-free water and incubating at $55^{\circ} \mathrm{C}$ with shaking at approx. $500 \mathrm{rpm}$ for $5 \mathrm{~min}$, after which the water was removed and $500 \mu \mathrm{l}$ Nexterion Hybridisation Buffer was added and incubated at $55^{\circ} \mathrm{C}$ for 5 min with shaking. The buffer was removed and the pre-heated PCR products were applied to the ArrayTubes and incubated at $55^{\circ} \mathrm{C}$ for $1 \mathrm{~h}$ with shaking at approx. $500 \mathrm{rpm}$. The ArrayTubes were washed three times: first in $2 \times$ SSC containing $0.01 \%$ Triton X-100 (Sigma-Aldrich, St Louis, MO, USA), then $2 \times$ SSC and finally in $0.2 \times \mathrm{SSC}$; for each wash step, the previous solution was removed, $500 \mu \mathrm{l}$ relevant wash buffer was added and the ArrayTubes were incubated for $5 \mathrm{~min}$ at room temperature with shaking at approx. $500 \mathrm{rpm}$. The final wash buffer was removed and $100 \mu$ l blocking solution containing $1 \times$ phosphate buffered saline (PBS), 2\% dried milk powder and $0.05 \%$ Triton X-100 was applied to each ArrayTube and incubated at $30^{\circ} \mathrm{C}$ for $15 \mathrm{~min}$, then removed. HRP-linked anti-biotin antibody (New England Biolabs, Ipswich, MA, USA) was diluted 1 in 100 in blocking solution and $100 \mu \mathrm{l}$ diluted antibody was applied to 
each array and incubated for $15 \mathrm{~min}$ at room temperature. Finally, the ArrayTubes were washed three times as described above in 1x PBS containing 0.1\% Tween 20 (Sigma) (500 $\mu \mathrm{l}$ for each wash step). The final wash buffer was removed and $100 \mu \mathrm{l}$ SeramunGrün Chip substrate (Seramun Diagnostica, Wolzig, Germany) was added to each ArrayTube. The ArrayTubes were scanned after approx. 15 20 min using an ATR-03 ArrayTube Reader (Alere), and images were analysed using the Iconoclust software package (Alere) as described by Cannon et al. [1].

\section{Results}

Effect of probe position on single base mismatch discrimination

Figures 2 and 3 shows typical results for arrays hybridised with PCR products (approximately $10^{12}$ copies per

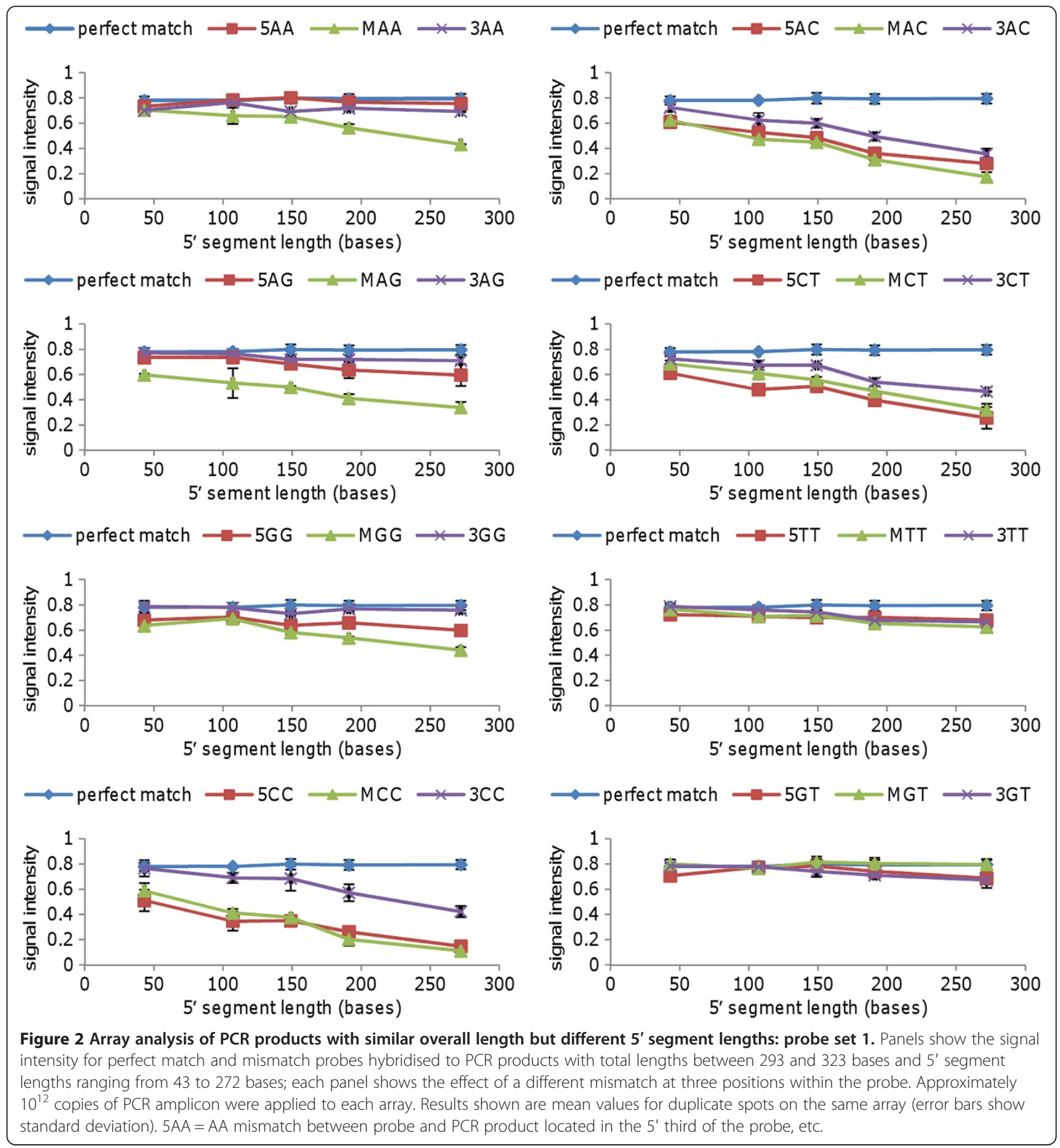


array) of similar total length (289 to 323 bases) but with $5^{\prime}$ segments ranging in length from 23 to 272 bases. Results are shown for eight different mismatches (AA, AC, AG, CT, GG, TT, CC and GT) in three different positions in the probe, as shown in Table 1. Similar signal intensities were observed for hybridisation to the perfect match probe, regardless of the probe position. Signal attenuation was observed for some mismatches in both probe sets (for example, AC and CC), while a GT mismatch was tolerated in both sets, and other mismatches (for example, AA and TT) were tolerated in the sequence context of one set but not the other. Mismatches located in the central section of the probe typically resulted in greater signal attentuation than equivalent mismatches located closer to the $3^{\prime}$ or $5^{\prime}$ end of the probe, in common with the results of others using various
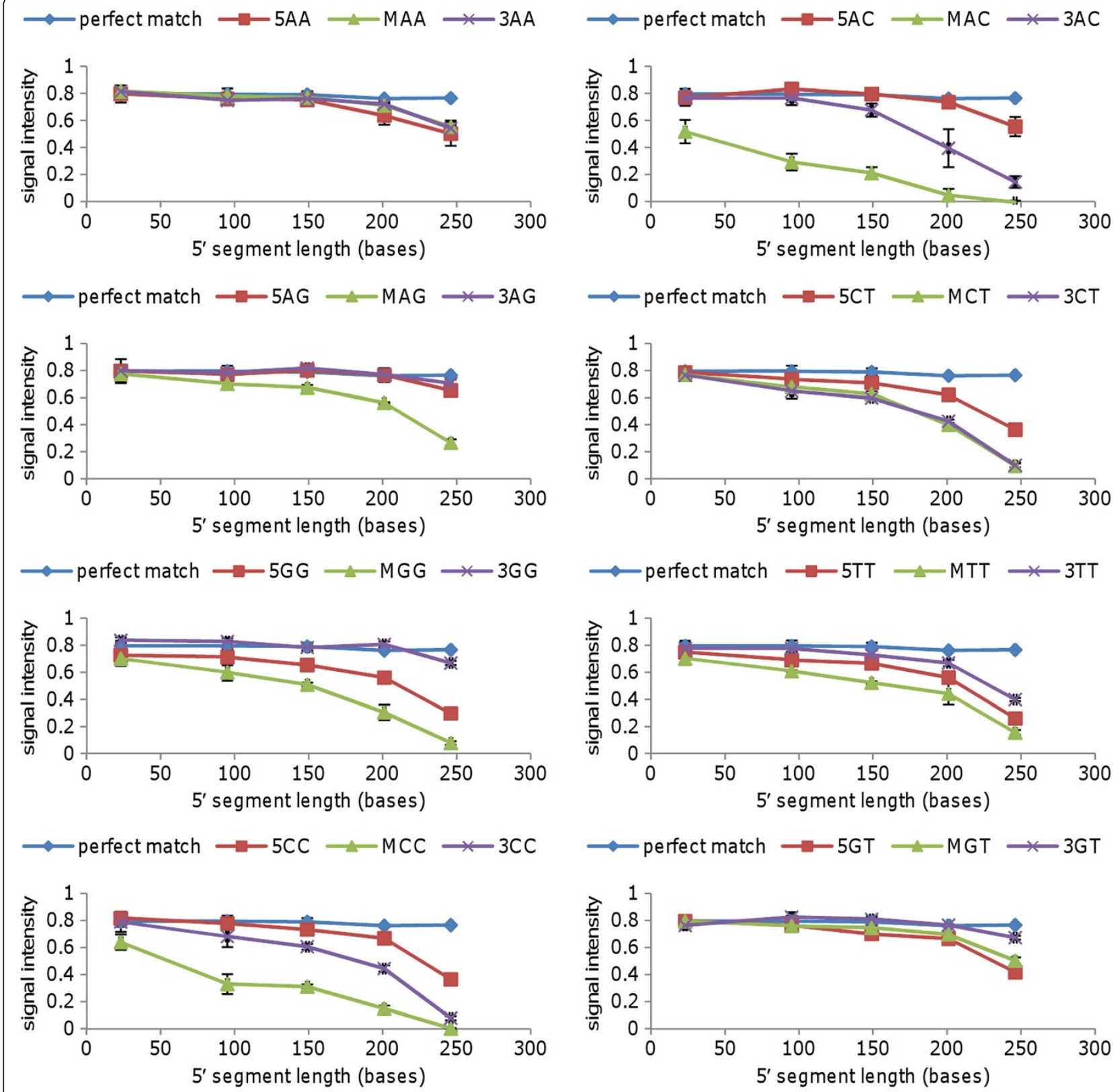

Figure 3 Array analysis of PCR products with similar overall length but different $5^{\prime}$ segment lengths: probe set 2. Panels show the signal intensity for perfect match and mismatch probes hybridised to PCR products with total lengths between 289 and 317 bases and $5^{\prime}$ segment lengths ranging from 23 to 246 bases; each panel shows the effect of a different mismatch at three positions within the probe. Approximately $10^{12}$ copies of PCR amplicon were applied to each array. Results shown are mean values for duplicate spots on the same array (error bars show standard deviation). 5AA = AA mismatch between probe and PCR product located in the $5^{\prime}$ third of the probe, etc. 
microarray platforms $[7,8,14]$. However, as shown in Figures 2 and 3, the degree of attenuation was observed to increase as the length of the $5^{\prime}$ segment of the PCR product (proximal to the array surface) was increased.

\section{Effect of probe position on limit of detection}

PCR products with similar total lengths but different $5^{\prime}$ segment lengths were diluted and hybridised to arrays to determine the limit of detection for hybridisation to perfect match probes. No difference in limit of detection was observed for a pair of PCR products with $5^{\prime}$ segment lengths of 43 and 272 hybridised to perfect match probe 1 , or for PCR products with $5^{\prime}$ segment lengths of 23 and 246 hybridised to perfect match probe 2 , as shown in Table 3.

\section{Combined effect of total product length and probe position}

Figure 4 shows typical results for groups of arrays hybridised with PCR products with the same $5^{\prime}$ segment length (43, 191 or 272 bases) and total lengths ranging from 94 to 978,242 to 1126 or 323 to 1207 bases, respectively. Note that results are shown only for mismatches located in the middle third of the probes. As in the previous experiment, a GT mismatch was tolerated while other mismatches caused a greater degree of signal attenuation. The greatest signal attenuation was observed for PCR products where the surface-proximal 5' segment constituted a larger proportion of the total PCR products; single base mismatches had a greater effect on signal intensity for short PCR products with a proportionally longer $5^{\prime}$ segment.

\section{Effect of primer ratio}

Figure 5A shows results for arrays hybridised with products of symmetrical PCR and asymmetrical PCR (primer ratio 1:5); similar results were observed for PCR products obtained using primer ratios of 1:10 and 1:20 (data not shown). Note that results are shown only for mismatches located in the middle third of the probes. The symmetrical and asymmetrical PCR products had total lengths of 312 and 317 bases, respectively, and 5' segment lengths of 43 bases. Figure 5B shows the symmetrical and asymmetrical PCR products analysed by agarose

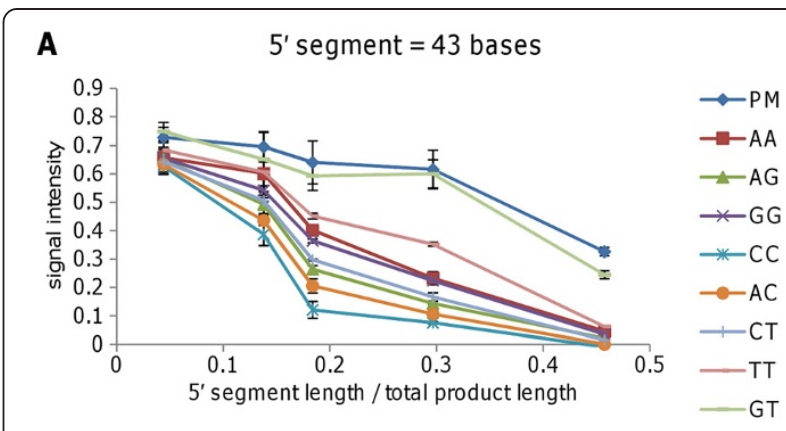

B $\quad 5^{\prime}$ segment $=191$ bases

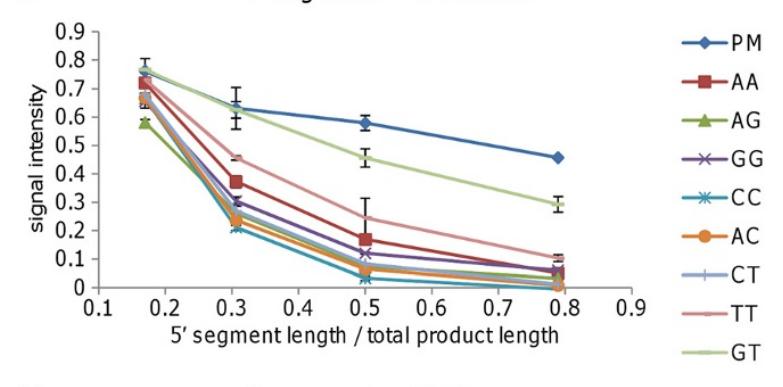

C $5^{\prime}$ segment $=272$ bases

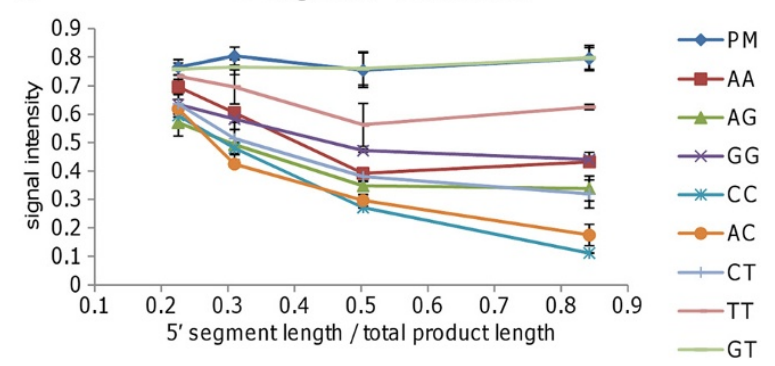

Figure 4 Array analysis of PCR products with the same 5' segment length but different total lengths. Arrays were hybridised with PCR products with constant 5 ' segment lengths (A: 43 bases; B: 191 bases; C: 272 bases) but different overall lengths ranging from 94 to 978 bases, 242 to 1126 bases, and 323 to 1207 bases, respectively. Signal intensities were normalised relative to the signal for the perfect match probe on each array and are plotted against the 5 ' segment length shown as a proportion of the total product length. Approximately $10^{12}$ copies of PCR amplicon were applied to each array. Results shown are mean values for duplicate spots on the same array (error bars show standard deviation). $\mathrm{PM}=$ perfect match probe. $\mathrm{AA}=\mathrm{AA}$ mismatch between probe and PCR product, etc.

Table 3 Limit of detection of PCR products with similar total lengths but different $\mathbf{5}^{\prime}$ segment lengths

\begin{tabular}{lllll}
\hline & & \multicolumn{2}{l}{ Signal intensity (mean $\pm \mathbf{s d}$ ) } \\
\cline { 3 - 5 } Total length (bases) & $\mathbf{5}^{\prime}$ segment length (bases) & Approx. $\mathbf{8} \times \mathbf{1 0}^{\mathbf{9}}$ copies & Approx. $\mathbf{8} \times \mathbf{1 0}^{\mathbf{8}}$ copies & Approx. $\mathbf{8} \times \mathbf{1 0}^{\mathbf{7}}$ copies \\
\hline 312 & 43 & $0.63 \pm 0.03$ & $0.23 \pm 0.03$ & - \\
323 & 272 & $0.62 \pm 0.01$ & $0.22 \pm 0.02$ & - \\
296 & 23 & $0.66 \pm 0.06$ & $0.28 \pm 0.05$ & - \\
317 & 246 & $0.57 \pm 0.03$ & $0.24 \pm 0.00$ & - \\
\hline
\end{tabular}

*Results shown are mean values for duplicate spots on the same array; -: negative. 


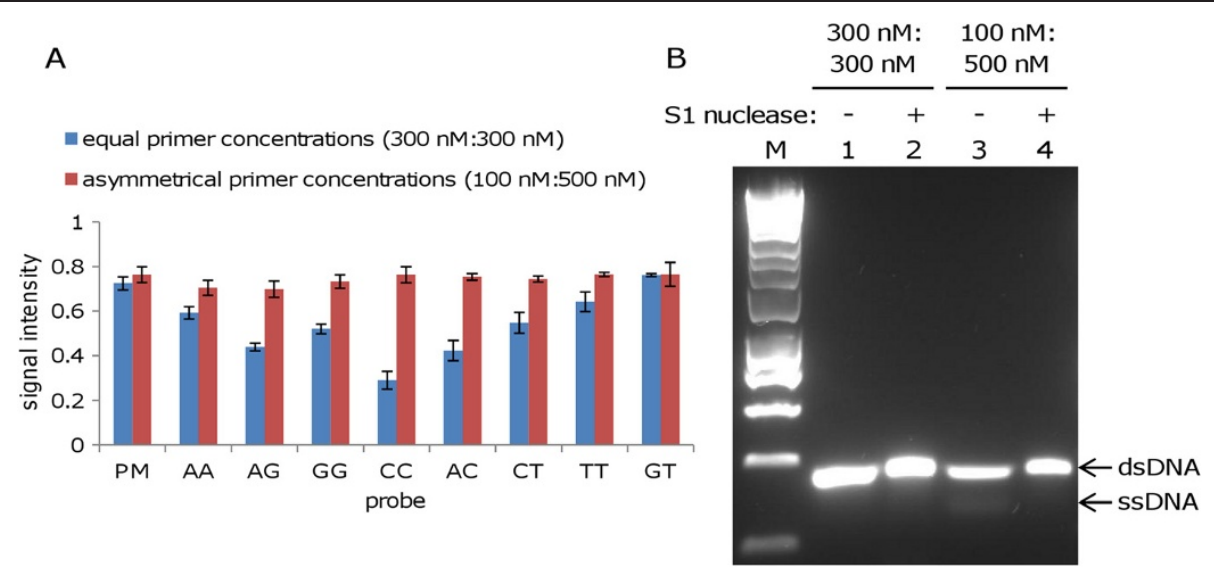

Figure 5 Array analysis of symmetrical and asymmetrical PCR products. A. Signal intensity for hybridisation of symmetrical and asymmetrical PCR products of 312 and 317 bases, respectively, both with a 5' segment length of 43 bases. Asymmetrical PCR was carried out using a forward primer modified for linear-after-the-exponential (LATE) PCR, with a primer ratio of 1:10. Approximately 500 ng total DNA (single-stranded plus double-stranded) was hybridised to each array. Results shown are mean values for duplicate spots on the same array. AA = AA mismatch between probe and PCR product, etc. B. Agarose gel electrophoresis of symmetrical and asymmetrical PCR products. The products of symmetrical and asymmetrical PCR were visualised by agarose gel electrophoresis with GelRed nucleic acid stain before and after treatment with S1 nuclease to confirm that the product of asymmetrical PCR contained single-stranded DNA (removed by S1 nuclease) as well as double-stranded DNA.

gel electrophoresis before and after treatment with S1 nuclease to demonstrate the presence of single-stranded DNA in the product generated by asymmetrical PCR. For the product of symmetrical PCR, signal attenuation was observed for some mismatches; however, similar signals were observed for the perfect match probe and all mismatched probes for the asymmetrical product.

\section{Discussion}

The ArrayTube hybridisation architecture in the format used here contains around 200 locations for the deposition of DNA probes depending on the spacing between spots. With two replicates of each probe and an appropriate multiplexed PCR this allows the detection/discrimination of around 80-90 targets and subtyping of panels of associated agents along with appropriate controls such as for DNA extraction. Since this is an open platform, the user has control over the number of targets and the specific properties of the probes being used. The technology can also be used in a strip format for the simultaneous processing of up to 96 arrays, potentially allowing the development of higher throughput screening capabilities than other multiplex PCR-based platforms [23].

Clinical diagnostic applications require methods with high sensitivity and robustness. By using an array format it is possible to screen a sample for multiple targets from the same agent (for example, plasmid and chromosomal sequences) $[3,24]$ and this redundancy has the potential to increase the robustness of detection in terms of the avoidance of both false positives and false negatives [24]. The ability to discriminate small sequence differences is crucial for some purposes, such as the discrimination of SNPs in the identification of antimicrobial resistance [25] or differentiation of bacterial genotypes [26]. Microarrays for pathogen detection are often designed to detect a relatively narrowly defined group of targets (for example, a particular bacterial taxon in a specified clinical matrix). More versatile arrays may be developed to perform a set of related functions, such as detection of a range of organisms and identification of the presence of antimicrobial resistance markers [4], or simultaneous detection of diverse agents, for example, bacteria and viruses, in the same sample. For the effective detection of viruses it may be necessary to tolerate multiple sequence differences, while for the detection of bacteria there may be a need to accurately discriminate between targets on the basis of minor sequence differences. While sensitivity will be largely influenced by PCR efficiency, our results indicate that it could be possible to leverage the specificity (or conversely, inclusivity) of detection by manipulating the length of the target's PCR amplicon, positioning the probe with respect to the $5^{\prime}$ end of the hybridising strand and changing the primer ratio to generate double-stranded or partially singlestranded products.

In line with previous reports, we observed that different mismatches were tolerated to different degrees, with the greatest effect when the mismatch was located close to the centre of the probe $[8,27,28]$. In the limited probe sets that we used, a GT mismatch was tolerated (little or no attenuation of the hybridisation signal) while $\mathrm{CC}, \mathrm{AC}$ and CT mismatches caused significant signal attenuation. However, we also observed that the same mismatched probe 
was observed to give different signal intensities when approximately equal numbers of copies of PCR products differing in terms of probe position were hybridised to the array. The maximum effect of single mismatches (and in particular CC, AC and CT mismatches) was observed for PCR products where a greater proportion of the total length was at the $5^{\prime}$ end relative to the probe (and therefore proximal to the array surface). A reduction in the discrimination of individual mismatches was observed if PCR primers were used at unequal concentrations resulting in generation of some single-stranded product, presumably because the complementary non-target strand competes with the shorter mismatched probe for hybridisation to the target strand. This is consistent with the proposal of Peytavi et al. [18] that the complementary strand destabilises the target-probe duplex, particularly in relation to the length of the distal segment of the hybridised target. We did not observe a reduction in signal strength with a longer 3' segment of the PCR product (distal to the array surface) as was reported by Peytavi et al. (in fact, as shown in Figure 4, signal strength decreased with a shorter 3' segment length); however, there are differences between the two experimental systems, including the probe orientation and hybridisation conditions, which could partially explain this difference. Our results suggest that in the experimental set-up used here, mismatch discrimination is most likely to be achieved using equal primer concentrations, while inclusive detection of sequence variants would be enhanced by the use of asymmetrical primer concentrations.

It should be noted that the results presented are for PCR using single primer pairs, and factors such as product length and primer ratio may impact on the performance of a primer pair in a multiplex reaction in terms of sensitivity. For example, shorter PCR amplicons may generally be preferable due to the likelihood of higher yields [29] and reduced formation of intra- and intermolecular structures which are reported to hinder hybridisation $[15,18,19]$.

\section{Conclusions}

Our results suggest a strategy for the rational design of PCR primers in order to leverage the exclusivity (specificity) or inclusivity of detection of individual targets on the array. Specifically, positioning a probe within the PCR product such that there is a longer section proximal to the array surface may increase the discrimination of single mismatches. As these primer design strategies could be applied independently to each target as required, this presents a means of fine-tuning an array for detection of potentially diverse targets. Optimisation of array performance by adjustment of the primers used for multiplex PCR has the potential to increase flexibility and cost effectiveness in comparison with redesigning the probes themselves.
Competing interests

The authors declare that they have no competing interests.

\section{Authors' contributions}

JT, SAW, NB, SAG conceived and designed the experiments; JT, CH performed the experiments and analyzed the data. JT, SAW, NB, CH, SAG contributed to drafting of the manuscript and read and approved the final manuscript.

\section{Acknowledgments}

This study was funded by the UK Ministry of Defence (Programme Office). The funding body approved submission of the manuscript for publication but had no role in study design, data collection and analysis, or preparation of the manuscript. The authors thank Lois Blackman and Matthias Dorsch of Defence Science Technology Organisation, Melbourne, Australia for the B. pseudomallei DNA used in this study.

\section{Author details}

${ }^{1}$ The Food and Environment Research Agency, Sand Hutton, YO41 1LZ York, UK. ${ }^{2}$ Defence Science and Technology Laboratory, Porton Down, SP4 0JQ Salisbury, UK.

Received: 13 July 2013 Accepted: 9 April 2014

Published: 17 April 2014

\section{References}

1. Cannon GA, Carr MJ, Yandle Z, Schaffer K, Kidney R, Hosny G, Doyle A, Ryan J, Gunson R, Collins T, Carman WF, Connell J, Hall WW: A low density oligonucleotide microarray for the detection of viral and atypical bacterial respiratory pathogens. J Virol Methods 2010, 163:17-24.

2. Felder KM, Hoelzle K, Wittenbrink MM, Zeder M, Ehricht R, Hoelzle LE: A DNA microarray facilitates the diagnosis of Bacillus anthracis in environmental samples. Lett Appl Microbiol 2009, 49:324-331.

3. Schmoock G, Ehricht R, Melzer F, Rassbach A, Scholz HC, Neubauer H, Sachse K, Mota RA, Sagib M, Elschner M: DNA microarray-based detection and identification of Burkholderia mallei, Burkholderia pseudomallei and Burkholderia spp. Mol Cell Probes 2009, 23:178-187.

4. Järvinen AK, Laakso S, Piiparinen P, Aittakorpi A, Lindfors M, Huopaniemi L, Piiparinen $H$, Mäki M: Rapid identification of bacterial pathogens using a PCR- and microarray-based assay. BMC Microbiol 2009, 9:161.

5. Wang D, Coscoy L, Zylberberg M, Avila PC, Boushey HA, Ganem D, DeRisi JL: Microarray-based detection and genotyping of viral pathogens. Proc Natl Acad Sci U S A 2002, 99:15687-15692.

6. Chou CC, Chen $\mathrm{CH}$, Lee $\Pi$, Peck K: Optimization of probe length and the number of probes per gene for optimal microarray analysis of gene expression. Nucleic Acids Res 2004, 32:e99.

7. Suzuki S, Ono N, Furusawa C, Kashiwagi A, Yomo T: Experimental optimization of probe length to increase the sequence specificity of high-density oligonucleotide microarrays. BMC Genomics 2007, 8:373.

8. Naiser T, Ehler O, Kayser J, Mai T, Michel W, Ott A: Impact of pointmutations on the affinity of surface-bound DNA/DNA and RNA/DNA oligonucleotide-duplexes: comparison of single base mismatches and base bulges. BMC Biotechnol 2008, 8:48

9. Karaman MW, Groshen S, Lee C-C, Pike BL, Hacia JG: Comparisons of substitution, insertion and deletion probes for resequencing and mutational analysis using oligonucleotide microarrays. Nucleic Acids Res 2005, 33:e33.

10. Southern E, Mir K, Shchepinov M: Molecular interactions on microarrays. Nat Genet 1999, 21:5-9.

11. Shchepinov MS, Case-Green SC, Southern EM: Steric factors influencing hybridisation of nucleic acids to oligonucleotide arrays. Nucleic Acids Res 1997, 25:1155-1161.

12. Letowski J, Brousseau R, Masson L: Designing better probes: effect of probe size, mismatch position and number on hybridization in DNA oligonucleotide microarrays. J Microbiol Methods 2004, 57:269-278.

13. Kolchinsky A, Mirzabekov A: Analysis of SNPs and other genomic variations using gel-based chips. Hum Mutat 2002, 19:343-360.

14. Hadiwikarta WW, Walter J-C, Hooyberghs J, Carlon E: Probing hybridization parameters from microarray experiments: nearest-neighbour model and beyond. Nucleic Acids Res 2012, 40:e138. 
15. Peplies J, Glöckner FO, Amann R: Optimization strategies for DNA microarray-based detection of bacteria with $16 \mathrm{~S}$ rRNA-targeting oligonucleotide probes. Appl Environ Microbiol 2003, 69:1397-1407.

16. Wei T, Pearson MN, Armstrong K, Blohm D, Liu J: Analysis of crucial factors resulting in microarray hybridization failure. Mol Biosyst 2012, 8:1325-1338.

17. Nguyen HK, Southern EM: Minimising the secondary structure of DNA targets by incorporation of a modified deoxynucleoside: implication for nucleic acid analysis by hybridisation. Nucleic Acids Res 2000, 28:3904-3909.

18. Peytavi R, Tang LY, Raymond FR, Boissinot K, Bissonnette L, Boissinot M, Picard FJ, Huletsky A, Ouellette M, Bergeron MG: Correlation between microarray DNA hybridization efficiency and the position of short capture probe on the target nucleic acid. Biotechniques 2005, 39:89-96.

19. Stedtfeld RD, Wick LM, Baushke SW, Tourlousse DM, Herzog AB, Xia Y, Rouillard JM, Klappenbach JA, Cole JR, Gulari E, Tiedje JM, Hashsham SA: Influence of dangling ends and surface-proximal tails of targets on probe-target duplex formation in 16S rRNA gene-based diagnostic arrays. Appl Environ Microbiol 2007, 73:380-389.

20. Guo Z, Guilfoyle RA, Thiel AJ, Wang R, Smith LM: Direct fluorescence analysis of genetic polymorphisms by hybridization with oligonucleotide arrays on glass supports. Nucleic Acids Res 1994, 11:5456-5465.

21. Pierce KE, Sanchez JA, Rice JE, Wangh LJ: Linear-after-the-exponential (LATE)-PCR: primer design criteria for high yields of specific singlestranded DNA and improved real-time detection. Proc Natl Acad Sci U S A 2005, 102:8609-8614.

22. Tang X, Morris SL, Langone JJ, Bockstahler LE: Simple and effective method for generating single-stranded DNA targets and probes. Biotechniques 2006, 40:759-763.

23. Weller SA, Cox V, Essex-Lopresti A, Hartley MG, Parsons TM, Rachwal PA, Stapleton HL, Lukaszewski RA: Evaluation of two multiplex real-time PCR screening capabilities for the detection of Bacillus anthracis, Francisella tularensis, and Yersinia pestis in blood samples generated from murine infection models. J Med Microbio/ 2012, 61:1546-1555.

24. Janse I, Bok JM, Hamidjaja RA, Hodemaekers HM, van Rotterdam BJ: Development and comparison of two assay formats for parallel detection of four biothreat pathogens by using suspension microarrays. PLoS One 2012, 7:e31958

25. Zhu LX, Zhang ZW, Liang D, Jiang D, Wang C, Du N, Zhang Q, Mitchelson K, Cheng J: Multiplex asymmetric PCR-based oligonucleotide microarray for detection of drug resistance genes containing single mutations in Enterobacteriaceae. Antimicrob Agents Chemother 2007, 51:3707-3713.

26. Ballarini A, Scalet G, Kos M, Cramer N, Wiehlmann L, Jousson O: Molecular typing and epidemiological investigation of clinical populations of Pseudomonas aeruginosa using an oligonucleotide-microarray. BMC Microbiol 2012, 12:152.

27. Urakawa H, El Fantroussi S, Smidt H, Smoot JC, Tribou EH, Kelly JJ, Noble PA, Stahl DA: Optimization of single-base-pair mismatch discrimination in oligonucleotide microarrays. Appl Environ Microbiol 2003, 69:2848-2856.

28. Gresham D, Dunham MJ, Botstein D: Comparing whole genomes using DNA microarrays. Nat Rev Genet 2008, 9:291-302.

29. Ehricht R, Slickers P, Goellner S, Hotzel H, Sachse K: Optimized DNA microarray assay allows detection and genotyping of single PCR-amplifiable target copies. Mol Cell Probes 2006, 20:60-63.

\section{Submit your next manuscript to BioMed Central and take full advantage of:}

- Convenient online submission

- Thorough peer review

- No space constraints or color figure charges

- Immediate publication on acceptance

- Inclusion in PubMed, CAS, Scopus and Google Scholar

- Research which is freely available for redistribution 